\title{
Item measurement for logistics-oriented belt conveyor systems using a scenario-driven approach and automata-based control design
}

\author{
Ho-min Park*†, Arnout Van Messem*‡,Wesley De Neve*† \\ ${ }^{*}$ Center for Biotech Data Science, Department of Environmental Technology, Food Technology \\ and Molecular Biotechnology, Ghent University Global Campus, Incheon, Korea \\ ${ }^{\dagger}$ IDLab, Department of Electronics and Information Systems, Ghent University, Ghent, Belgium \\ ${ }^{\ddagger}$ Department of Applied Mathematics, Computer Science and Statistics, Ghent University, Ghent, Belgium \\ \{homin.park, arnout.vanmessem,wesley.deneve\}@ghent.ac.kr
}

\begin{abstract}
Given the success of online shopping platforms and e-commerce technology, there is an increasing necessity to quickly and safely package different types of items. Addressing this necessity requires technology to accurately measure items at a high speed. Existing studies, however, lack in terms of reproducibility and the diversity of the items measured. In this paper, we present a novel approach for item measurement, targeting automated packaging systems that make use of belt conveyors. In particular, leveraging a scenario-driven approach and an automata-based control design, we describe in detail the creation of a real-world prototype for belt conveyor-based item measurement. Experimental results obtained for this prototype demonstrate that it is possible to measure different types of items (boxes, books, household items) with a mean error of less than $3.02 \mathrm{~mm}$ and a standard deviation of less than $2.34 \mathrm{~mm}$, for a maximum conveyor belt speed that is less than $0.5 \mathrm{~m} / \mathrm{s}$ and a maximum calculation time of $20 \mathrm{~ms}$.
\end{abstract}

Keywords-automata-based programming, belt conveyors, object-oriented design, sensor fusion, size measurement

\section{INTRODUCTION}

Imagine a logistics scenario in which a conveyor belt is rotating at a high speed, with items of random shape moving over this belt, one at a time and also at a certain distance from one another. Given this scenario, being able to accurately acquire the size of an item at a high speed is for instance useful for automated item packing and subsequent storage delivery. In this paper, we propose a solution for the problem of measuring the size of an item in the aforementioned scenario. By solving this problem, we aim at automating the detection and classification of items, and at real-time processing of the information obtained, without compromising the logistics throughput.

\section{A. Problem Statement}

Suppose $n$ sensors are attached to a belt conveyor. When an item passes by, it goes through the area monitored by the different sensors, with the $i$-th sensor producing a set of measurements $S_{i}=\left\{s_{j} \mid s_{j} \in \mathbb{R}, 1 \leq j \leq \gamma(i)\right\}$, and where $\gamma(i)$ denotes the number of sensor values emitted by the $i$-th sensor. In this study, we would like to create a sensor fusion function $f_{\text {fuse }}$, taking as input the set $I=\bigcup_{i=1}^{n} S_{i}$ and generating as output a tuple $O=(w, l, h)$, where $w, l, h \in \mathbb{R}$ specify a bounding box of width $w$, length $l$, and height $h$.

\section{B. Related Work}

Many attempts have already been made to automatically measure the size of items in a belt conveyor environment. We summarize a number of representative studies in Table I. The column 'Target shape' indicates the shape of the item that is transported by the conveyor. A number of measurement systems only target box-type or flat-top shapes, while other systems do not care about the shape of the items that need to be measured. The column 'Output' in Table I specifies the type of data generated by the measurement system. Typically, measurement systems return either the size of a bounding box, which is the size of the smallest cuboid surrounding the item at hand, or the volume of the item under consideration [1], [2]. The column '\# of test items' represents the number of items each study used for the purpose of performance evaluation. Furthermore, the column 'Equipment' summarizes the type of hardware used to implement the measurement system. Finally, the column 'Methods' outlines the core concept(s) used by the measurement system.

The authors of [3] focus on measuring the size of boxtype items that can be placed on a conveyor belt. They set up three 3-D cameras that look down on an object of interest, with each 3-D camera acquiring a depth map for the boxes at hand. A depth map is a kind of image that stores distance information instead of color information for a particular scene, making it possible to extract a 3-D point cloud by adopting coordinate mapping. Furthermore, the authors present a method to detect and calculate planes in the point cloud extracted, hereby using regression analysis to perform corrections for each plane.

In line with [3], the authors of [4] describe a boxbased measurement system, only using a single 3-D camera and setting it up from above. Moreover, their system does not utilize the entire depth map, but only a cross-shaped portion that is called a Region-Of-Interest (ROI). As a result, the calculation speed is significantly faster. In addition, 


\begin{tabular}{c|c|l|c|l|l|} 
Paper & Target shape & Output & $\begin{array}{c}\text { \# of test } \\
\text { items }\end{array}$ & \multicolumn{1}{|c|}{ Equipment } \\
\hline$[3]$ & Box & Bounding box & 3 & Three structured light cameras & 3-axis plane detection \\
{$[4]$} & Box & Bounding box & 5 & A time-of-flight camera & Cross line ROI, RANSAC \\
{$[1]$} & Any & Volume & - & A time-of-flight scanner & Trapezoid cross section \\
{$[2]$} & Any & Volume & - & A structured light laser & Average of distance differences \\
{$[5]$} & Flat-top items & Bounding box & 5 & A laser scanner, an encoder, an IR diode & Client-server structure \\
Proposed & Any & Bounding box & 8 & Two laser curtain sensors, an encoder & Sensor fusion, automata-based design
\end{tabular}

the RANSAC algorithm is used to mitigate point cloudbased errors. Finally, a design structure is proposed that hierarchically separates the part that acquires the ROI and the part that estimate the size of the boxes at hand.

The authors of [1] present a method for calculating the volume of wood chips moving on a conveyor belt using a scanner that is based on Time of Flight (ToF). The scanner has a 70 degree angle of view and a fan-shaped geometric measurement range. The authors present a method to concatenate the distance information measured by the scanner and to calculate the volume of the item at hand. The experiment was performed using a small and single cuboid.

The authors of [2] propose a method for determining the volume of an item by combining a laser and a monochrome camera. More precisely, the authors use a method to estimate a 3-D point cloud by using a laser irradiating the item of interest and a fixed camera monitoring the scene. The calibration method proposed by Zhengyou Zhang [6] was used to fix the intrinsic parameters of the camera and an experiment was performed to measure the volume of a cube with a side length of $100 \mathrm{~mm}$.

The authors of [5] focus on a more practical measurement system design. In order to flexibly cope with problems occurring in various environments, the authors propose a method for simultaneously collecting and processing data by adopting a client/server approach. The client is responsible for detecting items and measuring sensor values, and the server processes the values received from the client, calculating the actual rectangle contour and volume, and sending this information back to the client. A prototype was implemented using an Arduino compute platform and a laser scanner, and the effectiveness of the system was determined by measuring five flat-top items ten times each.

\section{Difference With Previous Research Efforts}

Unlike previous studies, this work focuses on the following aspects. First, we designed the proposed measurement system to be robust against the influence of different environmental conditions using sensor fusion. Second, we present a robust algorithm that enables the system to reliably measure items continuously coming in over a high-speed conveyor belt. Finally, the system we propose can measure various types of shapes, as empirically verified through a prototype implementation.
1) Robustness Against Different Environmental Conditions: Previous studies mostly use cameras or sensors with one focus. That way, it is easy to obtain a wide range of measurements at once, but these can be easily influenced by external environmental conditions such as light scattering, reflection, and occlusion [7]. If these models are to be applied in real-world industrial use cases, additional equipment is needed in order to minimize the resulting environmental errors. Instead, we propose to fuse two multi-focus light curtain sensors and a rotary encoder in order to minimize the influence of environmental conditions.

2) Algorithmic Exposition: While a number of studies have investigated the core aspects of a measurement system, such as [1], [2], [6], those studies lack specific details about the structure of the measurement system proposed. With this in mind, we have described our measurement system in a reproducible manner, along with the theoretical limits we calculated while building the sensor fusion system.

3) Measuring Various Types of Items: The approaches put forward in [3]-[5] can only measure items of limited geometry. For example, [3], [4] assumes the use of boxlike items only, and the algorithm of [5] assumes a flat top surface. As such, the aforementioned approaches are limited in terms of the types of items that can be measured. However, the prototype we present in this paper does not place any limitation on the shape of the items that need to processed. Specifically, we performed measurement experiments with typical box-shaped items, but also with various types of items, such as sauce pans, indoor shoes, and buckets. Given these measurement experiments, even though various items were used, an average error of at most $3.02 \mathrm{~mm}$ and a standard deviation of $2.34 \mathrm{~mm}$ is achieved. This is on par with the results of [3], [5], and better than the results presented in [4].

\section{Paper Organization}

Starting from Section III, we first explain a scenario in which a measurement system for belt conveyors is used, facilitating problem abstraction by conducting an objectoriented analysis. In addition, we propose an automata-based algorithm to perform fast and stable fusion of different sensor measurements. In Section IV, and based on the design outlined in Section III, we discuss the implementation of 
our measurement system. Next, in Section V, we infer the theoretical performance of the proposed system from the technical specifications of the devices used in our prototype implementation. We also present a comparative analysis of the practical performance of our prototype measurement system.

\section{BACKGROUND}

In this section, we summarize the concepts necessary to understand the proposed algorithm and the implemented prototype system. We start with explaining the software aspects, also gradually introducing several hardware aspects.

\section{A. Finite Automaton}

A finite-state machine (FSM) or finite automaton (FA) is a mathematical model used to design computer programs and electronic logic circuits. Both are sometimes simply referred to as a state machine. A finite automaton or an abstract machine can have a finite number of states. The machine can only be in one state at a time. A machine can change from one state to another by an event, which is called a transition. A particular finite automaton is defined by the set of possible states from the current state and the conditions that cause these transitions.

Automata-based programming is a way of designing a program by looking at the program, or part of it, as a finite state machine (FSM). This design approach simplifies and abstracts the complex logic that needs to be implemented into three components: states, events, and transitions. Systems designed by this programming technique recognize the given environment and determine their current state. These systems can then take action by performing a proper state transition [8], [9].

\section{B. Real-Time Operating Systems}

A Real-Time Operating System (RTOS) is an operating system developed for real-time applications, putting the focus on CPU time management features. Real-time operating systems give programmers more control over process priorities: the critical section of the system code is minimized and the priority of an application may exceed the priority of a system program. That way, it is possible to process an application request within a certain amount of time.

The main design goal of an RTOS is to ensure realtime performance, and not high throughput. An RTOS that generally meets its deadlines is called a soft real-time operating system, whereas an RTOS that meets such deadlines in a deterministic way is called a hard real-time operating system [10].

\section{Sensor Fusion}

Sensor fusion aims at combining data from various or multiple sensors in order to make the resulting information more reliable and accurate. The data used by a particular fusion approach do not necessarily have to originate from identical sensors. Data calibration is therefore often inevitable during fusion. Various ways of dealing with this have been suggested [11]-[14].

\section{Belt Conveyor Systems}

Belt conveyors, another term for belt conveyor systems, are systems that consist of two or more pulleys and a belt that connects these pulleys. One or more pulleys are powered, moving the belt and the items on the belt forward. Such systems are key to efficient cargo handling in manufacturing facilities and warehouses. [15]

\section{E. Light Curtain Sensors}

A light curtain sensor or area sensor is a multi-focus sensor that detects an abnormality in the area it monitors, typically using a transmitter and a receiver that work in pairs. This type of sensor uses the principle of linearity of light and modulation [16]. This means that a transmitter and a receiver share only a fixed signal; they do not affect nearby transceivers. The signals, which may take the form of laser light [17] or infrared light [18], can be used to detect the presence or location of an item, by monitoring the reception status of the receiver.

Light curtain sensors are mainly used for security and safety purposes in industrial sites. For example, they can be used to detect external intrusions to important systems [19] or they can be used for anomaly detection in production and logistics processes [20].

\section{F. Rotary Encoders}

Rotary encoders are sensors that allow tracking the rotation of a motor shaft to generate digital information such as position and movement [21], [22]. They usually realize position control for automation equipment, industrial equipment, and robots. Rotary encoders may contain specific form factors (ruggedness), durability, and resolution.

Among the different types of rotary encoders available, optical methods are the most widely used, leveraging an LED light source, a photo detector, and a code disk. The disc has holes along its perimeter. As the disk rotates, the led light passes through a hole. The photo detector detects this and passes current (up pulse). On the contrary, if there is no light passing through, no current is passed (down pulse). The encoder has two rows of holes on the disc. The two signals shown (A, B) are designed to cross each other, as shown in Figure 1. The direction of rotation can be derived from the order in which A and B occur. Counting can be done by setting the criteria for pulse evaluation. For example, if Eval has a value of 1, then counting happens when the pulse of either $\mathrm{A}$ or $\mathrm{B}$ ( $\mathrm{A}$ in the figure) moves up. If Eval has a value of 2 , then counting happens whenever the pulse of A moves up or down. Finally, if Eval has a value of 4, then counting happens whenever the pulse of A or B moves up or down. Section IV-B and Section V-A will pay more attention to the usage of the Eval parameter. 


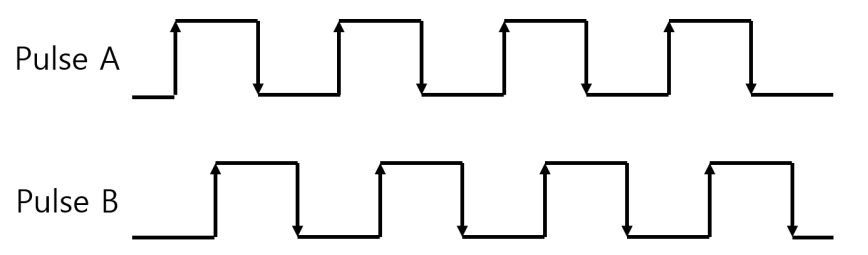

Eval $10 \times 11 \times 2 \times 3 \times 4$

Eval $20 \times 1 \times 2 \times 3 \times 4 \times 5 \times 6 \times 7 \times 8$

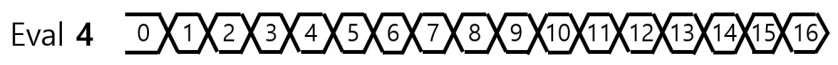

Figure 1. The signal of an encoder and its evaluation (clockwise)

\section{MethodS}

\section{A. Scenario Description and Abstraction}

To produce the fusion function $f_{\text {fuse }}$ as mentioned in Section I-A, we first need to make a number of assumptions about the environment in which this function works. Suppose we have a system with a conveyor belt that moves at a constant speed, with sensors deployed as shown in Figure 2. Unlike the authors of [3]-[5], who assume the use of boxshaped items or flat-top items of various sizes, in this study, we assume that the conveyor can move forward items of any type. For example, as shown in Figure 2, our measurement system may have to deal with a moving frying pan. As illustrated in Figure 3, the proposed measurement system scans a moving item in the red, green, and blue areas in order to find a cuboid of minimum size that contains the moving item under consideration. However, while doing so, the belt conveyor system should not be stopped for reasons of energy and time efficiency.

Using object-oriented design principles, we can make abstraction of our application scenario, as shown in Figure 4. The black squares represent objects (e.g., system parts), the black arrows denote messages, and the blue arrows with the round starting points are the arguments passed with each message. The "Target System" is a generic operating system that can command the "Conveyor Belt" to move and retrieve a minimum cuboid from the "Measurement System".

As an item passes through the areas that are being monitored, the measurement system fuses data and generates a minimum cuboid. In other words, the width, the height, and the length of the item passing through get measured, leading to the creation of a minimum cuboid. This minimum cuboid is transferred by the measurement system to the target system. The "Conveyor Belt" is a passive object associated with the target system, transporting the item placed on the belt. The "Minimum Cuboid" is a passive object created by the "Measurement System". It stores the measured 3-D size of the items that have crossed the scanning area.

Measurement begins when the "Target System" requests a measurement start signal. The "Target System" sends a move item request to the "Conveyor Belt". The item then passes over the belt. The "Target System" subsequently sends a make cuboid request to the "Measurement System", for the item currently transported by the conveyor system. The "Measurement System" receives the request and generates the "Minimum Cuboid" through the generate command and delivers it to the "Target System". The "Target System" can then utilize the "Minimum Cuboid" object created according to its application needs.

\section{B. Automata-Based Measurement Algorithm}

In this section, we describe the measurement algorithm used by the "Measurement System" shown in Figure 4. Borrowing from the paradigm of "automata-based programming", as introduced earlier in Section II-A, we also provide more algorithmic details in Figure 5. The algorithm consists of five states and nine transitions. The initial state Init and the special state Error are marked in blue and red, respectively. Also, the transitions to the error state are marked in red.

The start state of our algorithm is Init, which is in the upper left of Figure 5. In this state, the variables needed for measuring are initialized and constant values are set. When the initialization is complete, the "Measurement System" transitions to the Stable state through transition $t_{1}$. In the Stable state, the system stays idle using the self-transition $t_{2}$ until a random item reaches the scan area. When an item is near the scan area, the system detects it and enters the Measure state via $t_{3}$. In the Measure state, item size information is acquired using various sensors connected to the system. The acquisition of information continues until the item leaves the scan area through the self-transition $t_{4}$. When an item leaves the scan area, the system moves to the Calc state via $t_{5}$. In this state, the acquired "Minimum Cuboid" is created and delivered to the "Target System". After that, the system returns to the Stable state, again waiting to perform size measurements through $t_{6}$.

Problems can sometimes arise with any status. We deal with these problems by defining additional states. Whenever a problem occurs, the problem is solved by moving to the Error state through the $t_{e 1}$ and $t_{e 2}$ transitions. Upon correction of a problem, the system uses the $t_{e 3}$ transition to get back to the Stable state.

\section{PRototype System IMPlementation}

Given the explanatory notes in Section III, we implemented a prototype system for item size measurement. This section describes the different parts used by our prototype system, as well as the internal algorithms used.

\section{A. Hardware Description}

The hardware of the overall system is shown in Figure 6. Figure 6(a) shows a picture taken from the left side of our hardware setup, whereas Figure 6(b) shows a picture taken 


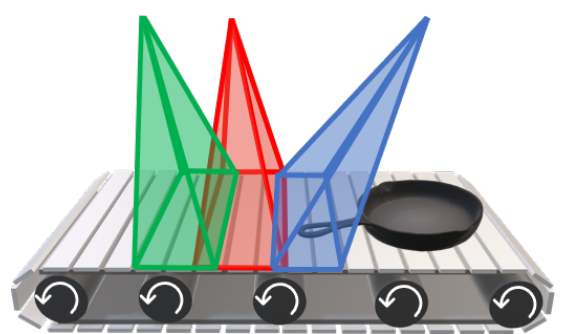

(a) Time 1

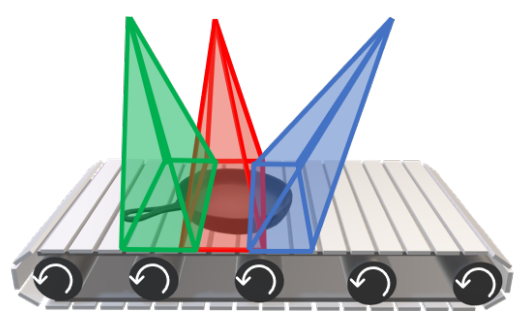

(b) Time 2

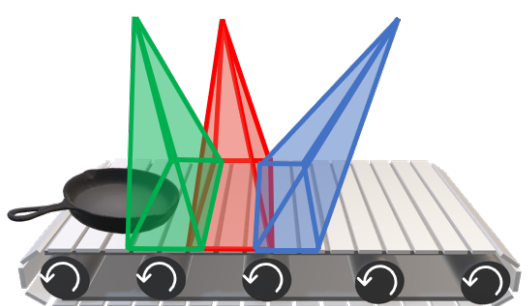

(c) Time 3

Figure 2. Sensor fusion for a belt conveyor system
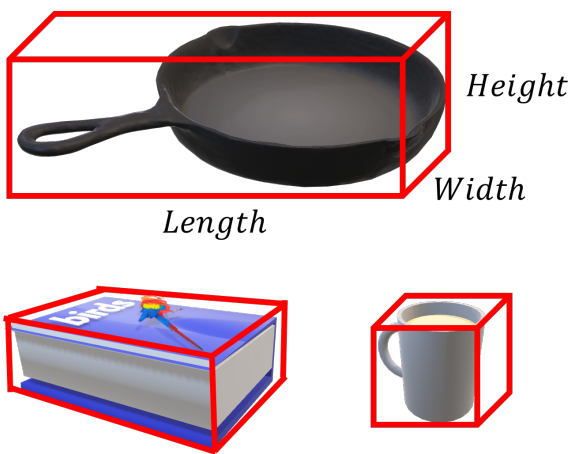

Figure 3. Examples of different minimum bounding cuboids

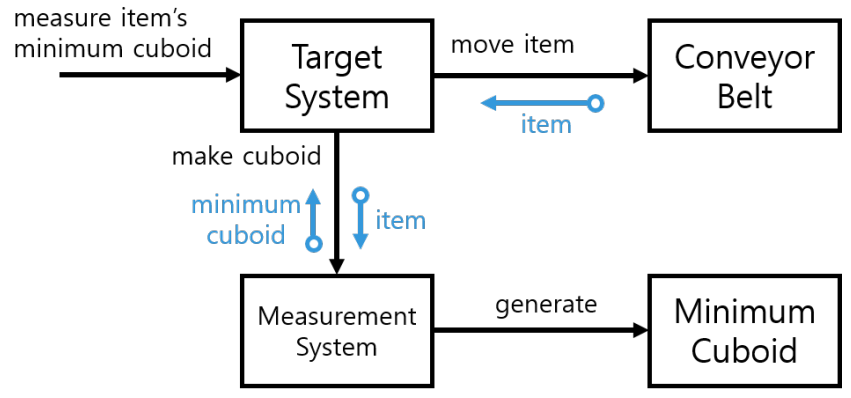

Figure 4. Object-oriented abstraction of our application scenario

from the right side, rotating the hardware over 180 degrees. The flat green area is a conveyor belt made of rubber, with the white arrow indicating the movement direction of the items. The red dashed lines parallel to each other visualize the curtain sensors, with the arrows indicating how the light travels from the transmitter to the parallel receiver. If an item passes, then the receiver cannot detect the laser beams associated with the parts covered by the item, making it possible to perform size calculations. In Figure 6(a), the laser curtain shown is installed up and down, making it possible to perform height measurements, and in Figure 6(b), the laser curtain is installed left and right, making it possible to perform width measurements. The yellow arrow in the lower-right corner of Figure 6(a) points to the rotary encoder, which is directly attached to the

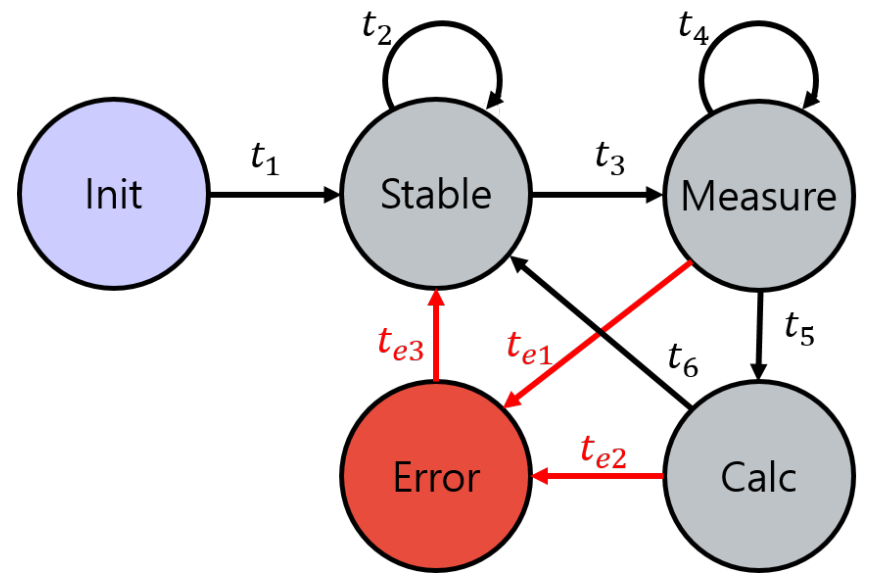

Figure 5. Automata-based measurement algorithm

motor shaft. The rotary encoder is responsible for sensing the rotation of the conveyor, as well as for performing length measurements. The dark purple attachment shown in the lower-right corner of Figure 6(b) shows the motor for driving the conveyor belt.

The system built has a gap in-between the two conveyor belts and moves with one drive shaft (motor). We use two pairs of laser curtain sensors. One pair is inserted in-between the two conveyor belts in order to measure the width of an item. The other pair is attached vertically to each side in order to measure the height of an item. The type of laser curtain sensor used is Leuze's CML720i series. We use an Autonics E40S6 Rotary Encoder with a resolution of 2000 , which means that rotations can be detected from $1 / 2000 \times 2 \pi$ revolutions. The embedded computer used is B\&R's X20CP1382. The Structured Text (ST) language [23] is used for programming, which is one of the five programming languages specified in IEC61131-3 [24].

\section{B. Pseudo Code and Parameter Setting}

We integrated the automata-based algorithm previously discussed in Section III-B into our prototype system. However, as X20CP1382 is a hard ROTS, there is a strict run-time limitation. If all of the given commands cannot be executed within a specified amount of time, then the OS will crash. As 


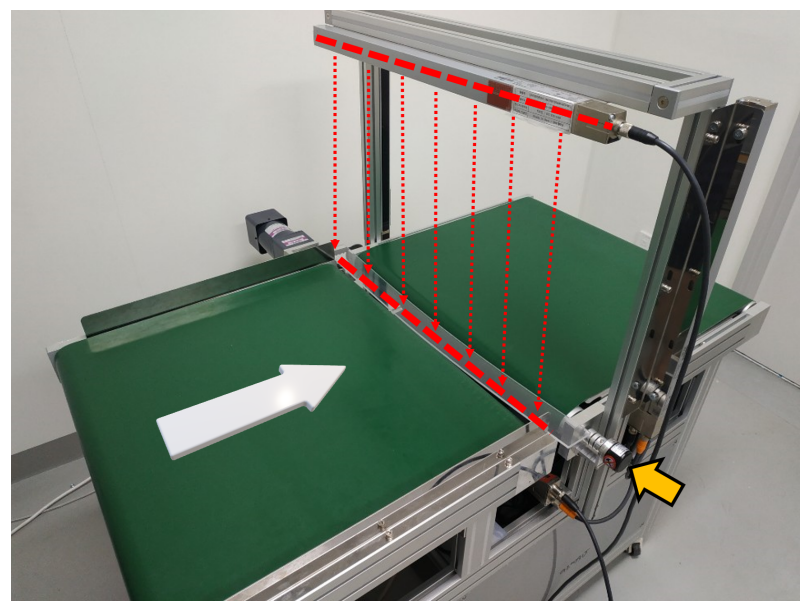

(a) Main direction

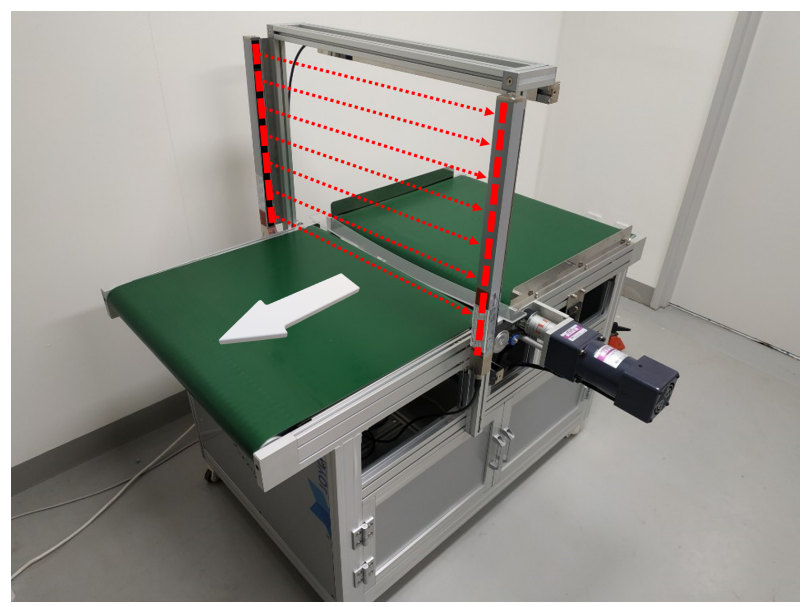

(b) Opposite direction

Figure 6. Prototype hardware overview

a result, to enhance the stability of our measurement system, we divided our automata-based algorithm into Algorithm 1, which is in charge of the Stable and Measure state, and Algorithm 2, which is in charge of the Calc and Error state. The former is a a high-priority process that needs a short cycle time for dealing with the sensor data produced. The latter is a low-priority process that can come with a long cycle time, given that this process is responsible for $\mathrm{I} / \mathrm{O}$ and communication with external functions. The two algorithms also operate as independent processes, with the exception of a shared storage space for variables.

Algorithm 1 is run as the highest priority process with a cycle time of about $10 \mathrm{~ms}$. The top line represents the data generated by each sensor: $w_{i n}$ and $h_{i n}$ refer to the values produced by the curtain sensors that are located in Figure 6(a) and Figure 6(b) each, and encoder en $_{\text {refers }}$ to the values produced by the encoder. Line 1 represents the Initial state, initializing the variables and constants needed to run the algorithm. $\epsilon$, which is by default set to $15 \mathrm{~mm}$, is the minimum sensor sensitivity needed to transition from the Stable state to the Measure state. $\tau$, which is a rotary encoder parameter, is defined by the following equation:

$$
\tau=\frac{1}{\frac{2 \pi r}{r e s \times e v a l}},
$$

with $2 \pi r$ representing the radius of the cross section of the conveyor belt pulley and with res denoting the resolution of the encoder attached to the pulley. eval refers to the way the encoder counts, as illustrated by Figure 1.

Intuitively speaking, tau is the number of encoder pulses required for the conveyor belt to move $1 \mathrm{~mm}$. For our conveyor belt, the outer radius is $32 \mathrm{~mm}(r)$, res $=2,000$, and eval $=4$. So, in theory, $\tau=39.7$. However, given the use of a real-world test environment, the conveyor belt needed additional pulses to move $1 \mathrm{~mm}$. Therefore, the parameter $\tau$ was adjusted and finally set to a value of 42.5 . This parameter is used to calculate the length of an item. $\tau_{\max }$ denotes the maximum length and is used as a criterion for transitioning to an error state when an item is too long. $w_{\max }$ and $h_{\max }$ are variables used to indicate the maximum width and the maximum height of an item, respectively. The state variable stable is set to 1 , denoting the state stable.

After initialization, the algorithm repeats the transition $t_{2}$ to the Stable state (i.e., state $=1, w_{\text {in }}<\epsilon$ ). This can be seen as an idle state in which no item is approaching.

Lines 3-7 implement a conditional statement that determines whether or not to transition to the Measure state when the system is in the Stable state. In particular, this conditional statement checks whether an item needs to be measured $\left(w_{i n}>\epsilon\right)$. In other words, this conditional statement checks whether or not the sensor for measuring the width in Figure 6(a) is triggered. After an item has been detected, the two variables $w_{\max }$ and $h_{\max }$ required for the measurement are initialized to zero.

Lines 8-15 show the $t_{4}$ transition, which saves the values in the Measure state. In $t_{4}$, the system stores the maximum height and width of the item.

Lines 16-18 show the $t_{5}$ transition to the $\mathrm{Cal}$ c state upon completion of the measurements. The Calc state is located in Algorithm 2.

Lines 19-22 are used to trigger $t_{e 1}$ when a new item has been detected during the execution of Algorithm 2. In this case, the system moves to the Error state (state $=5$ ).

Lines 23-26 contain the statements to invoke $t_{e 2}$. For example, when the item to be measured in the Measure state is too long $(30,000 / 42.5 \approx 700 \mathrm{~mm})$, then a switch to the Error state occurs.

Algorithm 2 is executed with a cycle time of $100 \mathrm{~ms}$. The variables in Algorithm 1 and Algorithm 2 are shared. 
Lines 3-9 of Algorithm 2 are responsible for organizing and sending the measurement results (that is, the process of moving each measured value to an output variable), where $l_{\text {out }}$ stores the length $(\mathrm{mm})$ of an item, as obtained by dividing the total number of encoder pulses by $\tau$. The values of $w_{\text {out }}, h_{\text {out }}$, and $l_{\text {out }}$ thus obtained are passed to the target system as objects through a separate communication function send_cuboid_dims().

Finally, we set the state variable to 1 in order to switch to the Stable state. Lines 10-15 execute the function error_handling () in order to deal with error conditions. In our prototype, the warning LED is then set to blink.

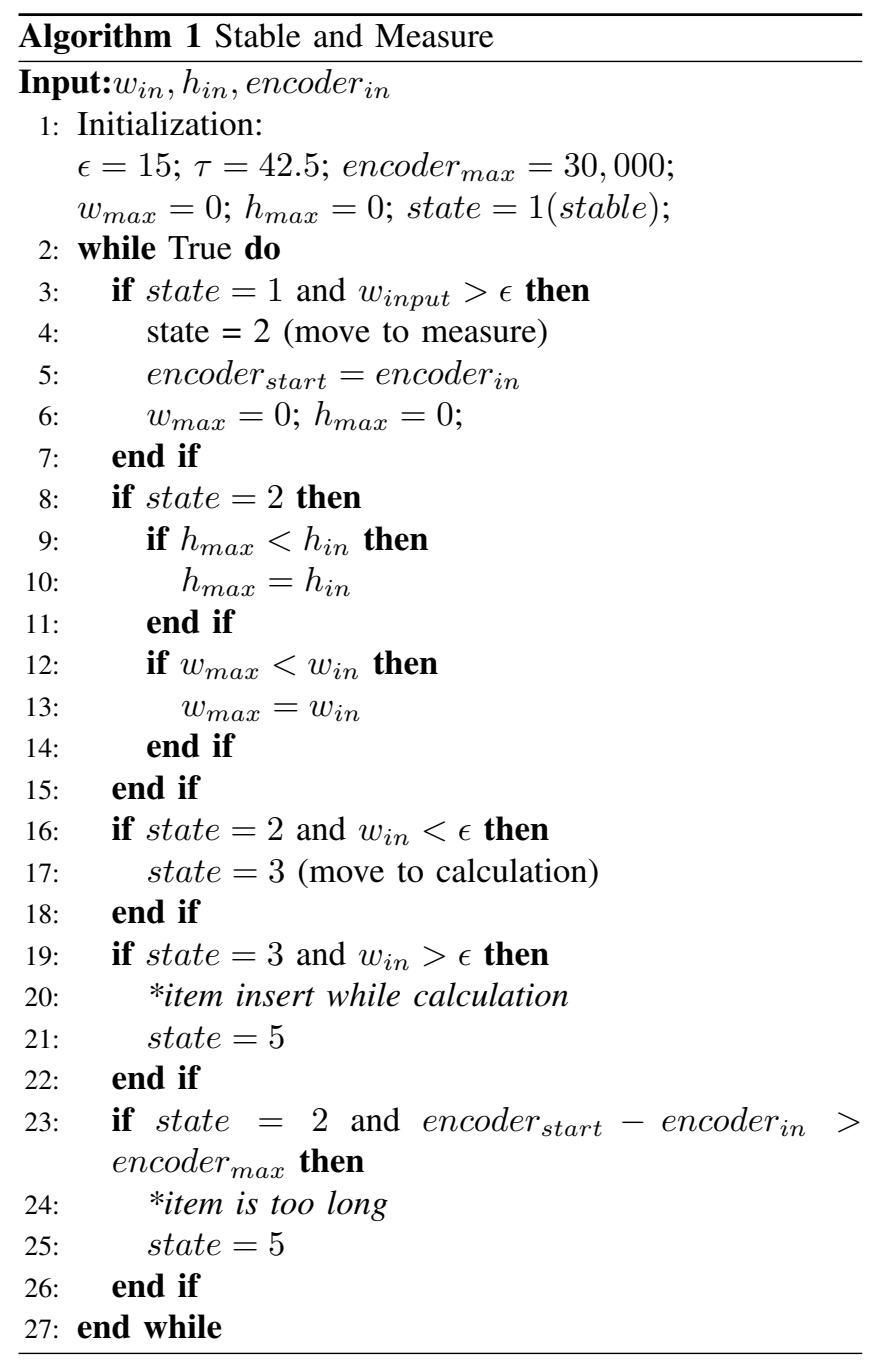

\section{EXPERIMENTS}

We conducted a number of experiments to evaluate the performance of our system. First, we estimate the theoretical performance of our system based on information available in the data sheets of the hardware used. Second, we evaluate the practical performance of our system by measuring eight samples 35 times, following the principle that if the number

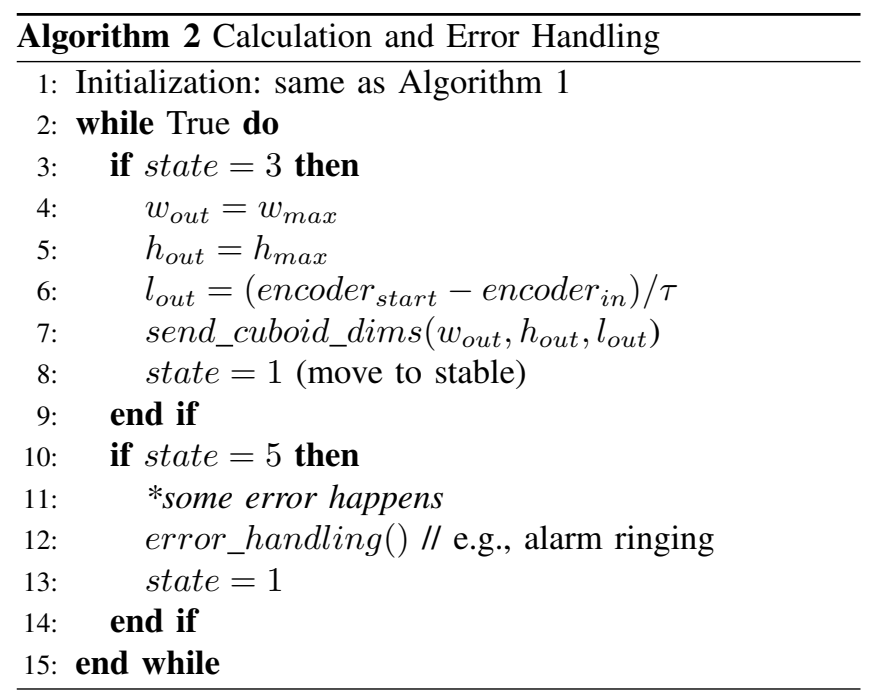

of samples is large enough (more than 30 times), the sample distribution will approximate the normal distribution, given the central limit theorem [25].

\section{A. Theoretical Performance}

In terms of maximum measurement performance, our prototype system can measure items that are up to $640 \mathrm{~mm}$ wide, $475 \mathrm{~mm}$ high, and approximately $700 \mathrm{~mm}$ long. These limitations are imposed by the sensors used and the value of the variable encoder $_{\max }$ in Algorithm 1. The measurement resolution of the system is $5 \mathrm{~mm}$ for the width and the height. This measurement resolution is affected by the characteristics of the laser curtain sensors. The resolution of the rotary encoder is $1 / 8000$, as defined by the specification of the rotary encoder and the evaluation method shown in Figure 1. The cycle time of a laser curtain sensor is number_of_beams $\times 0.03 \mathrm{~ms}+0.4 \mathrm{~ms}$. The sensors used in our prototype system have 128 width sensors and 96 height sensors. Therefore, each has a cycle time of 3.28 and $4.24 \mathrm{~ms}$. In case of the embedded computer, the width and height signals are scanned in $0.1 \mathrm{~ms}$ time. However, since the processor reading this value executes a cycle with a time of $10 \mathrm{~ms}$, once every $10 \mathrm{~ms}$, the maximum time for the algorithm to detect the presence of an item is $10 \mathrm{~ms}$. This maximum delay influences the error caused by the conveyor speed when measuring the length of an item. This influence is given as follows:

$$
L_{e r r}=v \times t_{e r r}+\epsilon,
$$

with $L_{e r r}$ denoting the length error, $v$ being the speed at which the item is moving (that is, $v$ is the speed of the conveyor belt), $t$ denoting the maximum delay, and $\epsilon$ being a small random number. Given this equation, at a conveyor belt speed of $0.5 \mathrm{~m} / \mathrm{s}$, a maximum delay of $0.01 \mathrm{~s}(10 \mathrm{~ms})$ can cause an error of $5 \mathrm{~mm}+\epsilon$ at worst. In other words, the 
deviation of the length can be up to $5 \mathrm{~mm}$. If the speed is faster, then the error will linearly increase.

\section{B. Practical Performance}

For evaluating the practical performance of the proposed measurement system, we prepared eight items that can be transported by the belt conveyor used. Figure 7 shows the way each item looks like: the items $\mathrm{A}$ to $\mathrm{C}$ denote three types of shipping boxes, as commonly used by parcel delivery services; the items D and E are two books; and the complex items $\mathrm{F}$ to $\mathrm{H}$ are assumed to have a random shape ( $\mathrm{F}$ is a saucepan, $\mathrm{G}$ is a round bucket, and $\mathrm{H}$ is an indoor shoe). The width, height, and length of the minimum bounding cuboids surrounding each item were measured with a vernier caliper and a tape measure. These measurements can be found in the ground truth columns of Table II. We ran a total of 35 measurement experiments at a conveyor speed of $0.5 \mathrm{~m} / \mathrm{s}$, with the size calculation taking $20 \mathrm{~ms}$ once an item has completely passed through the sensing area $(20 \mathrm{~ms}$ is the cycle time allotted to Algorithm 2.

We summarize the measurement results obtained in Table II. In general, the standard deviation is less than $2.65 \mathrm{~mm}$ along the different dimensions, with $\mathrm{F}$ and $\mathrm{G}$ being the only items to come with a standard deviation less than $4.99 \mathrm{~mm}$. The average measurement error is less than $3.02 \mathrm{~mm}$ overall, and the measurement errors obtained for the books, which are uniform in size and weight, tend to be very small. Of the complex items, $\mathrm{F}$ and $\mathrm{G}$ came with small measurement errors along the width dimension, given that the smooth transport of these two items was hampered by the gap in the belt conveyor, and where this gap is needed for sensor placement. However, the standard deviation for both items is still within $4.99 \mathrm{~mm}$. The gap in the belt conveyor can possibly be overcome through the placement of a flat transparent panel, still allowing for sensor light coming through.

Figure 8 plots the standard deviation as a function of the conveyor belt velocity, dividing the eight items into three classes: Boxes, Books, and Complex Items. In general, we can observe that the standard deviation decreases linearly as a function of the conveyor belt speed. This can be attributed to the fact that the cycle time and the resolution of the rotary encoder, as discussed in Section V-A, are affected by the conveyor belt speed.

\section{Discussion}

In this section, we compare the performance of our approach with the performance of approaches described in previous studies. However, given that previous studies were conducted in various environments, the different performance results obtained are only provided as a reference for the interested reader. Furthermore, given the experiments conducted by previous studies, we tried to increase the trustworthiness of our experimental results by increasing the number of measurements performed per item ( 35 times). The

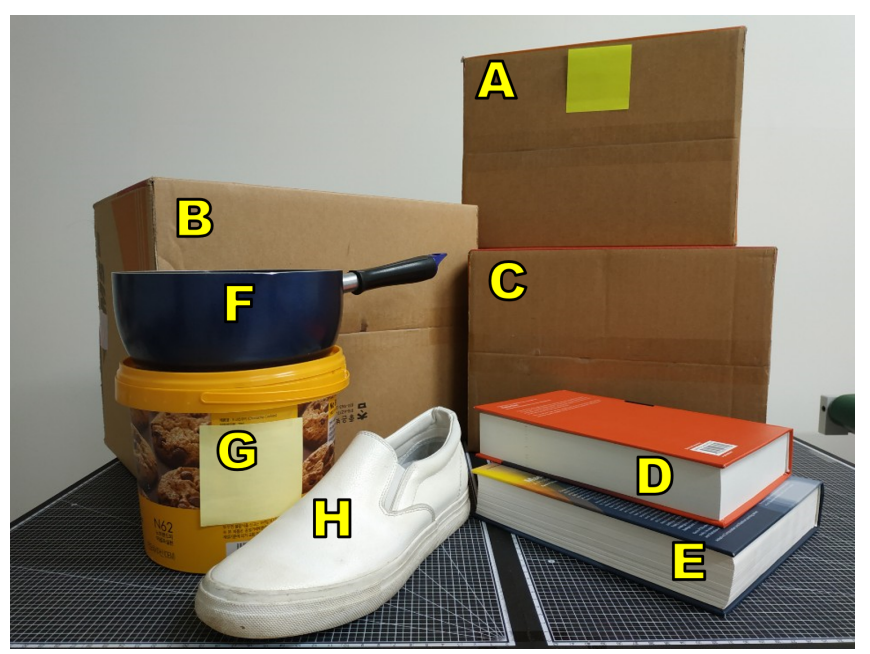

Figure 7. Items used in our measurement experiments: A to E are boxshaped items whereas $\mathrm{F}$ to $\mathrm{H}$ are irregularly shaped items

experimental setup used by our experiments is summarized in Table III. Note that Table III does not pay attention to the previously mentioned studies [1], [2] because a detailed description of the experimental setup and the measured items is not available.

In Table III, "\# of items used" represents the number of items used in the experiment and "Volume of largest item $\left(\mathrm{m}^{3}\right)$ " represents the volume of the largest item used. Table III contains the latter column as it gives a rough idea of the maximum measurement performance of the system. "Maximum speed" is the maximum speed of the conveyor belt. "Computation time (ms)" is the number of milliseconds needed to calculate the bounding box. "\# of measurements per item" is the number of experiments performed for each item. The latter number affects the reliability of the reported measurement results. Among the aforementioned indicators, our prototype system shows excellent results in terms of "\# of items", "Computation time (ms)", and "\# of measurements per item".

Table IV summarizes the mean error obtained by each study. The prototype we presented is the best in terms of length measurement, showing an error of less than $3 \mathrm{~mm}$ on average, even though it included many kinds of items and also irregularly shaped items. In Table V, we summarize the standard deviation reported by each study. We can see that the total standard deviation obtained by the proposed approach is within $2.34 \mathrm{~mm}$, even though we made use of a significant number of trials and various types of items.

\section{CONCLUSIONS AND FUtURE WORK}

In this paper, we introduced a novel approach for belt conveyor-based item measurement, using a three-step refinement process to design this approach. First, we discussed our application scenario on moving item measurement for belt conveyors and its corresponding requirements. Second, 


\begin{tabular}{|c|c|c|c|c|c|c|}
\hline \multirow{2}{*}{$\begin{array}{l}\text { Item name } \\
\text { (Description) }\end{array}$} & \multicolumn{2}{|r|}{ Width (mm) } & \multicolumn{2}{|c|}{ Height $(\mathrm{mm})$} & \multicolumn{2}{|c|}{ Length $(\mathrm{mm})$} \\
\hline & Ground truth & Estimated (mean \pm std) & Ground truth & Estimated (mean \pm std) & Ground truth & Estimated (mean \pm std) \\
\hline A (Box type 1) & 259.54 & $257.12 \pm 2.50$ & 105.92 & $105.62 \pm 1.67$ & 349.00 & $352.66 \pm 2.50$ \\
\hline B (Box type 2) & 257.48 & $261.62 \pm 2.37$ & 213.58 & $214.37 \pm 1.67$ & 347.00 & $346.97 \pm 2.37$ \\
\hline C (Box type 3) & 320.00 & $324.87 \pm 2.65$ & 284.93 & $285.75 \pm 1.80$ & 422.00 & $423.16 \pm 2.73$ \\
\hline D (Red book) & 162.01 & $164.25 \pm 1.80$ & 46.22 & $46.25 \pm 2.19$ & 239.10 & $239.05 \pm 2.50$ \\
\hline E (Blue book) & 209.23 & $210.50 \pm 1.51$ & 48.12 & $45.25 \pm 1.10$ & 237.08 & $237.15 \pm 2.84$ \\
\hline F (Saucepan) & 185.48 & $183.37 \pm 2.37$ & 92.49 & $111.37 \pm 3.39$ & 317.00 & $324.07 \pm 2.46$ \\
\hline G (Bucket) & 190.06 & $189.62 \pm 1.74$ & 162.00 & $171.75 \pm 3.49$ & 191.03 & $197.43 \pm 4.99$ \\
\hline H (Shoe) & 105.85 & $104.87 \pm 0.79$ & 85.78 & $84.00 \pm 2.02$ & 289.57 & $289.81 \pm 2.67$ \\
\hline
\end{tabular}

Table II

ITEM MEASUREMENT RESULTS

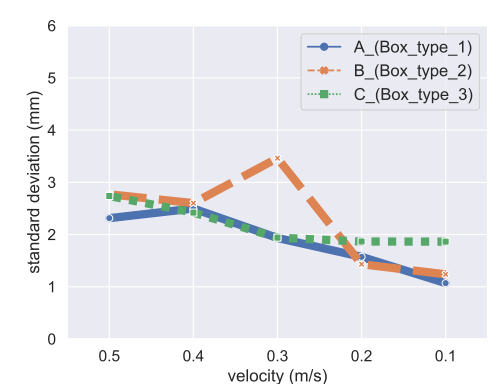

(a) Boxes

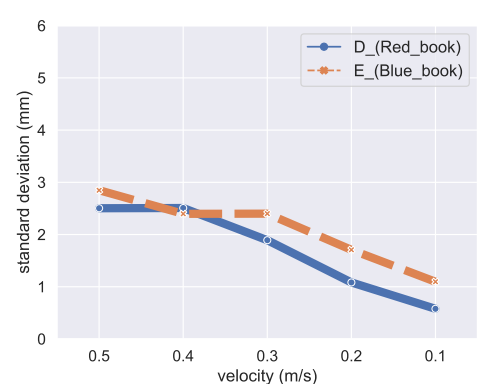

(b) Books

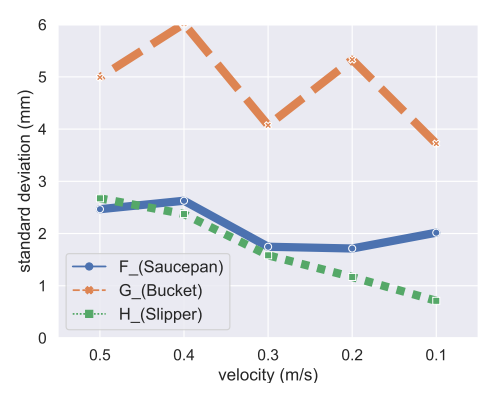

(c) Complex items

Figure 8. Standard deviation as a function of conveyor velocity

\begin{tabular}{c|c|c|c|c|} 
& Proposed & {$[3]$} & {$[4]$} & {$[5]$} \\
\hline \# of items used & 8 (Figure 7) & 3 (boxes only) & 5 (boxes only) & 4 (boxes and cylinders) \\
Volume of largest item $\left(\mathrm{m}^{3}\right)$ & 0.04 & 0.08 & 0.04 & 0.0005 \\
Maximum conveyor speed & 0.5 & 0.9 & 0.9 & 0.2 \\
Computation time (ms) & 20 & 1,530 & 100 & - \\
\# of experiments per item & 35 & 18 & 10 & 10
\end{tabular}

Table III

COMPARISON OF THE EXPERIMENTAL SETUP USED BY THE DIFFERENT MEASUREMENT APPROACHES

\begin{tabular}{c|c|c|c|c|} 
& Width & Length & Height & Total \\
\hline Proposed (w/o jammed item) & 2.57 & 1.75 & 3.63 & 2.65 \\
Proposed (all items) & 2.3 & 2.33 & 4.4 & 3.02 \\
[3] & - & - & 1.7 & 2.61 \\
[4] & 4.48 & 4.63 & 2.93 & 4.01 \\
[5] & 2.1 & 2.9 & 1.2 & 2.05
\end{tabular}

Table IV

COMPARISON OF THE AVERAGE ERROR

\begin{tabular}{c|c|c|c|c} 
& Width & Length & Height & Total \\
\hline Proposed (w/o jammed item) & 1.99 & 1.97 & 2.58 & 2.19 \\
Proposed (all items) & 1.96 & 2.16 & 2.88 & 2.34 \\
[3] & - & - & 1.21 & 2.24 \\
[4] & 3.85 & 4.75 & 0.58 & 3.06 \\
[5] & 3.00 & 1.70 & 1.46 & 2.05
\end{tabular}

Table $\mathrm{V}$

COMPARISON OF THE STANDARD DEVIATION OF THE MEASUREMENT ERROR

given the targeted application scenario, we executed an object-oriented abstraction process. Third, given the obtained abstraction, we designed an automata-based algorithm for performing item measurement.

Furthermore, given the approach put forward, we built a prototype system, integrating different pieces of hardware and our automata-based algorithm. For generalization purposes, we also used eight items of various shapes in our measurement experiments. In addition, each item was measured 35 times in order to improve the reliability of the measurements generated.

Our experimental results show that the proposed system is able to measure the size of items with an average measurement error of less than $3.02 \mathrm{~mm}$. Although this is not the best performance among previous studies, we would like to make not that our experimental conditions are more challenging, including the use of items with various shapes. Furthermore, the time needed to perform size calculations is only $20 \mathrm{~ms}$, which makes our approach the fastest.

In future work, we plan to further improve the proposed system so that it is possible to perform size measurement for items that are even more complex. 


\section{ACKNOWLEDGMENT}

The research and development activities described in this paper were funded by Ghent University Global Campus (GUGC) and MOTIE (Ministry Of Trade, Industry, and Energy), Korea, under the Advanced Technology Center programme supervised by KEIT (Korea Evaluation Institute of Industrial Technology) (10077285, Development of a smart and automated packaging system for variable-type and variable-size products).

\section{REFERENCES}

[1] D. Fojtík, "Measurement of the volume of material on the conveyor belt measuring of the volume of wood chips during transport on the conveyor belt using a laser scanning," in Proceedings of the 2014 15th International Carpathian Control Conference (ICCC). IEEE, 2014, pp. 121-124.

[2] J. Li, G. Liu, and Y. Liu, "A dynamic volume measurement system with structured light vision," in 2016 31st Youth Academic Annual Conference of Chinese Association of Automation (YAC). IEEE, 2016, pp. 251-255.

[3] Y. Hamamura and B. Irie, "The measurement of carried cartons using multiple kinect sensors," in Proceeding of MVA2013 IAPR international conference on machine vision applications, 2013.

[4] H.-m. Park, A. Van Messem, and W. De Neve, "Boxscan: An efficient and effective algorithm for box dimension measurement in conveyor systems using a single RGB-D camera," in 7th IIAE International conference on Industrial Application Engineering (ICIAE 2019). The Institute of Industrial Applications Engineers (IIAE), 2019, pp. 160-167.

[5] K.-T. Song, S.-Q. Ou et al., "A client-server architecture for object volume measurement on a conveyor belt," in 2019 12th Asian Control Conference (ASCC). IEEE, 2019, pp. 901906.

[6] Z. Zhang, "A flexible new technique for camera calibration," IEEE Transactions on pattern analysis and machine intelligence, vol. 22, 2000.

[7] P. Zanuttigh, G. Marin, C. Dal Mutto, F. Dominio, L. Minto, and G. M. Cortelazzo, "Time-of-flight and structured light depth cameras," Technology and Applications, ISSBN, pp. 978-3, 2016.

[8] B. Yartsev, G. Korneev, A. Shalyto, and V. Kotov, "Automatabased programming of the reactive multi-agent control systems," in International Conference on Integration of Knowledge Intensive Multi-Agent Systems, 2005. IEEE, 2005, pp. 449-453.

[9] N. Polikarpova and A. Shalyto, "Automata-based programming," Piter (in Russian), 2009.

[10] A. Silberschatz, P. B. Galvin, and G. Gagne, "Operating system concepts. 9th," p. 283-305, 2012.

[11] F. Shahdib, M. W. U. Bhuiyan, M. K. Hasan, and H. Mahmud, "Obstacle detection and object size measurement for autonomous mobile robot using sensor," International Journal of Computer Applications, vol. 66, no. 9, 2013.
[12] M. Bahrepour, N. Meratnia, Z. Taghikhaki, and P. J. Havinga, "Sensor fusion-based activity recognition for parkinson patients," Sensor Fusion-Foundation and Applications, pp. 171190, 2011.

[13] J. N. Gross, Y. Gu, M. B. Rhudy, S. Gururajan, and M. R. Napolitano, "Flight-test evaluation of sensor fusion algorithms for attitude estimation," IEEE Transactions on Aerospace and Electronic Systems, vol. 48, no. 3, pp. 2128 2139, 2012.

[14] V. Joshi, N. Rajamani, T. Katsuki, N. Prathapaneni, and L. V. Subramaniam, "Information fusion based learning for frugal traffic state sensing," in Twenty-Third International Joint Conference on Artificial Intelligence, 2013.

[15] P. M. McGuire, Conveyors: application, selection, and integration. CRC Press, 2009.

[16] W. W. Arrasmith, Systems engineering and analysis of electro-optical and infrared systems. CRC Press, 2018.

[17] P. Bartu, A. Neulinger, B. Jakoby, S. Bauer, and R. Koeppe, "Light curtain for 2D large-area object detection," Optics express, vol. 21, no. 10, pp. 12 757-12 766, 2013.

[18] J. J. García, J. Ureña, M. Mazo, and Á. Hernández, "Ir barrier data integration for obstacle detection," in Sensor and Data Fusion. IntechOpen, 2009.

[19] M. Gilewski, "Optical curtain transceiver," in 2018 26th Telecommunications Forum (TELFOR). IEEE, 2018, pp. 420-425.

[20] L. electronics. (2019, dec) cml720i measuring light curtain. [Online]. Available: http://bitly.kr/9oY3yS3

[21] J. Billingsley, A. Ellin, and G. Dolsak, "The design and application of rotary encoders," Sensor Review, 2008.

[22] E. Eitel, "Basics of rotary encoders: Overview and new technologies," Machine Design Magazine, vol. 7, 2014.

[23] T. Antonsen, PLC Controls with Structured Text (ST): IEC 61131-3 and best practice ST programming, 062018.

[24] M. Tiegelkamp and K.-H. John, IEC 61131-3: Programming industrial automation systems. Springer, 1995.

[25] S. G. Kwak and J. H. Kim, "Central limit theorem: the cornerstone of modern statistics," Korean journal of anesthesiology, vol. 70, no. 2, p. 144, 2017. 